\title{
MFAP5 facilitates the aggressiveness of intrahepatic Cholangiocarcinoma by activating the Notch1 signaling pathway
}

Jian-Hui Li ${ }^{1 \dagger}$, Xiao-Xu Zhu ${ }^{1 \dagger}$, Fu-Xi Li ${ }^{2}$, Chen-Song Huang ${ }^{1}$, Xi-Tai Huang ${ }^{1}$, Jie-Qin Wang ${ }^{1}$, Zhuo-Xing Gao ${ }^{2}$, Shi-Jin Li ${ }^{1}$, Qiong-Cong Xu', Wei Zhao ${ }^{2^{*}}$ and Xiao-Yu Yin ${ }^{1^{*}}$

\begin{abstract}
Background: Intrahepatic cholangiocarcinoma (ICC) is the second most common primary liver cancer. The dismal outcome of ICC patients is due to lack of early diagnosis, the aggressive biological behavior of ICC and the lack of effective therapeutic options. Early diagnosis and prognosis of ICC by non-invasive methods would be helpful in providing valuable information and developing effective treatment strategies.

Methods: Expression of microfibrillar-associated protein 5 (MFAP5) in the serum of ICC patients was detected by ELISA. Human ICC specimens were immunostained by MFAP5 antibodies. The growth rate of human ICC cell lines treated with MFAP5 or MFAP5 shRNAs was examined by CCK8 and colony formation assays. Cell cycle analysis was performed with PI staining. The effect of MFAP5 inhibition was assessed by xenograft models in nude mice. RNAseq and ATAC-seq analyses were used to dissect the molecular mechanism by which MFAP5 promoted ICC aggressiveness.

Results: We identified MFAP5 as a biomarker for the diagnosis and prognosis of ICC. Upregulated MFAP5 is a common feature in aggressive ICC patients' tissues. Importantly, MFAP5 level in the serum of ICC patients and healthy individuals showed significant differential expression profiles. Furthermore, we showed that MFAP5 promoted ICC cell growth and G1 to S-phase transition. Using RNA-seq expression and ATAC-seq chromatin accessibility profiling of ICC cells with suppressed MFAP5 secretion, we showed that MFAP5 regulated the expression of genes involved in the Notch1 signaling pathway. Furthermore, FLI-06, a Notch signaling inhibitor, completely abolished the MFAP5-dependent transcriptional programs.
\end{abstract}

Conclusions: Raised MFAP5 serum level is useful for differentiating ICC patients from healthy individuals, and could be helpful in ICC diagnosis, prognosis and therapies.

Keywords: MFAP5, Intrahepatic cholangiocarcinoma, Cancer biomarkers, NOTCH, Cell cycle arrest

\section{Background}

Intrahepatic cholangiocarcinoma (ICC) is a highly aggressive and molecularly heterogeneous tumor arising from the epithelial cells of segmental or proximal branches of bile duct. ICC ranks as the second most lethal malignancies worldwide and accounts for $10-15 \%$ of all primary liver malignancies. Over the last 10-20

\footnotetext{
* Correspondence: zhaowei23@mail.sysu.edu.cn; yinxy@mail.sysu.edu.cn

${ }^{\dagger}$ Jian-Hui Li and Xiao-Xu Zhu contributed equally to this work.

${ }^{2}$ Key Laboratory of Stem Cells and Tissue Engineering (Sun Yat-Sen

University), Ministry of Education, Guangzhou 510080, China

'Department of Pancreato-Biliary Surgery, The First Affiliated Hospital of Sun Yat-sen University, Guangzhou 510080, China
}

years, the rapidly increasing incidence and high mortality rates of ICC globally become the focus of concern among clinicians $[1,2]$. Diagnosis of ICC is traditionally based on radiologic, serologic, and pathologic evaluations, but early diagnosis of ICC by non-invasive methods remains a great challenge [3]. Thus, ICC are largely diagnosed at a non-curable metastatic stage. Moreover, owing to the aggressive feature of ICC tumors, the postoperative prognosis of ICC patients is far from satisfying [4]. The recurrence rates of ICC after surgery are up to $79.5 \%$ [5]. There is urgent need to seek more effective diagnostic/prognosis biomarkers and

C The Author(s). 2019 Open Access This article is distributed under the terms of the Creative Commons Attribution 4.0 International License (http://creativecommons.org/licenses/by/4.0/), which permits unrestricted use, distribution, and 
targeted approaches for managing this challenging liver cancer.

The extracellular matrix (ECM) is a highly dynamic structure of noncellular components, including structural proteins (predominantly collagens), matricellular proteins [e.g., periostin, thrombospondins, osteopontin and secreted protein acidic and rich in cysteine (SPARC)] [6], proteoglycans, glycoproteins, and polysaccharides [7]. ECM not only provides structural and biochemical support to tumor tissue, but also remodels the tumor microenvironment leading to tumor progression acceleration and resistance to therapy. ECM molecules are also released into the bloodstream and might represent as biomarkers of tumor development. There is a growing body of evidence that hypersecretion of ECM proteins (e.g., periostin, tenascin-C) has also been associated with poor prognosis in patients following surgical resection of ICC [8]. A better understanding of how ECM remodeling affects ICC progression will contribute to the development of new diagnosis/prognosis markers and therapeutics.

Microfibrillar-associated protein 5 (MFAP5) is an ECM glycoprotein, and a component of microfibrils of the ECM that function in tissue development. MFAP5 secreted by mesenchymal stroma cells plays an essential role in hematopoiesis and immune systems. Loss function of MFAP5 inhibits bone loss in mice, whereas MFAP5 mutation is associated with the pathology of thoracic aortic aneurysms and dissections in human [9]. In addition, MFAP5 is crucial in regulating tumor progression in breast cancer, ovarian cancer and tongue cancer $[10,11]$. In ovarian cancer, cancer-associated fibroblasts-derived MFAP5 upregulates lipoma-preferred partner (LPP) gene to enhance the efficacy of chemotherapy [12]. Depletion of MFAP5 by siRNA significantly decreases ovarian tumor growth and metastasis [13]. Moreover, in human cholangiocarcinoma (CCA), YAP signaling activated MFAP5 secretion to promote tube formation of human microvascular endothelial cells [14]. However, the role of MFAP5 in ICC remains unclear.

In the present study, we identify MFAP5 as a useful serum biomarker in the diagnosis of ICC. MFAP5 promotes ICC cell growth and cell cycle transition through activation NOTCH pathway. Thus, NOTCH inhibitors may represent effective regents to block MFAP5 mediated ICC cell outgrowth.

\section{Materials and methods}

\section{Patients' specimens}

240 ICC patients, who had been diagnosed by histology and underwent radical hepatectomy at the First Affiliated Hospital of Sun Yat-sen University in Guangzhou China, were included in this study. The inclusion criteria were as follows: [1] histologically diagnosed ICC [2]; underwent radical hepatectomy [3]; survived longer than 30 days after hepatectomy [4]; had integrated clinicopathological data and follow-up data. Patients who met one of the following criteria were excluded from the study: [1] diagnosed perihilar cholangiocarcinoma (Klatskin tumor) [2]; with tumors mixed of HCC and ICC [3]; R1 or R2 resection or laparotomy with tumor biopsy [4]; had received neoadjuvant chemotherapy or radiotherapy before hepatectomy. 208 ICC patients underwent hepatectomy from January 2007 to June 2016 were recruited for immunohistochemistry assay and prognostic analysis. 40 ICC patients who underwent hepatectomy from September 2016 to October 2019 were recruited for immunohistochemistry assay, qRT-PCR assay and ELISA assay (32 patients were recruited for immunohistochemistry assay in Fig. 1d, qRT-PCR assay in Fig. 1c and ELISA assay in Fig. 2b; another 8 patients were recruited for ELISA assay in Fig. 2e). 8 healthy people and 13 hepatocellular carcinoma $(\mathrm{HCC})$ patients were recruited for ELISA assay (Fig. 2b). Clinicopathological characteristics of ICC patients were shown in Additional file 1: Table S1. This study was approved by the Ethical Committee of the First Affiliated Hospital of Sun Yat-sen University [15].

\section{Extraction and processing of gene expression omnibus (GEO)}

One Affymetrix Human Transcriptome Array [HTA-2 0] dataset (GSE76297) and one Illumina human Ref-8 v2.0 expression dataset (GSE26566) were selected. The raw data was downloaded from GEO database (https:// www.ncbi.nlm.nih.gov/gds/). The array data of GSE76297 included 92 CCA tissue samples and 92 noncancerous tissue samples. The dataset of GSE26566 consisted of 103 CCA tissue samples and 59 non-cancerous tissue samples. The specific analysis method had been described previously [16].

\section{Immunohistochemical (IHC) staining}

This staining assay was performed as described previously [17]. Slides containing the sections were stained with commercially available anti-MFAP5 (1:100,\#ab283028,Abcam) and anti-Ki-67 (1:100, \#ab156956,Abcam) antibodies. Staining intensity (negative, 0 ; mild, 1 ; moderate, 2 ; severe, 3 ) and proportion of positive cells (negative,0; $\leq 10 \%, 1 ;>$ 10 and $\leq 33 \%, 2$; $>33$ and $\leq 66 \%, 3$; $>66 \%, 4$ ) were quantified respectively. Two experienced pathologists scored the stained tissues independently.

\section{Enzyme-linked immunosorbent assay (ELISA)}

The expression level of MFAP5 in ICC patients'serum was evaluated using ELISA kit (SEF590Hu Cloud-Clone Corp). The collected serum was all derived from preoperative clinical diagnosis of ICC patients. 


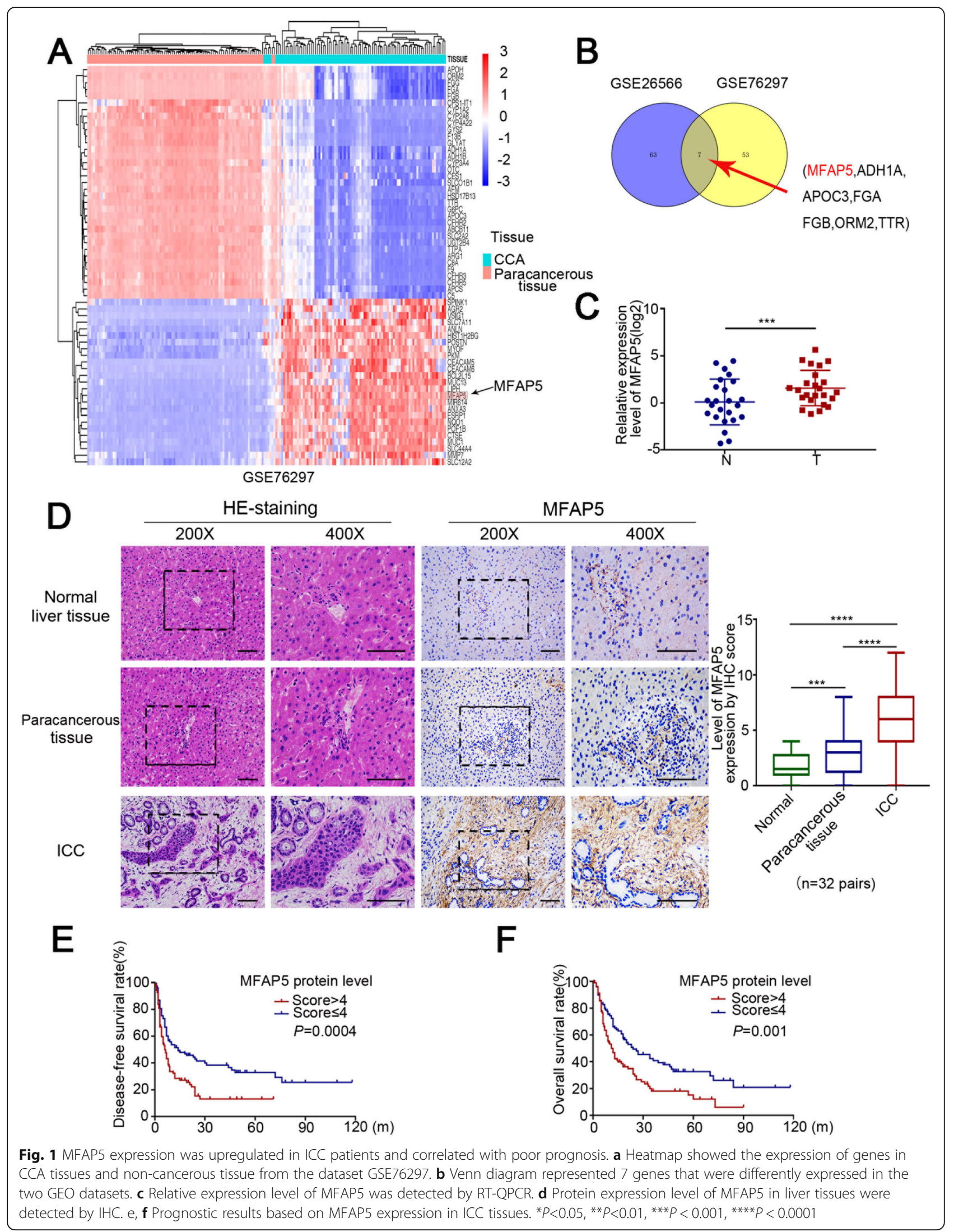




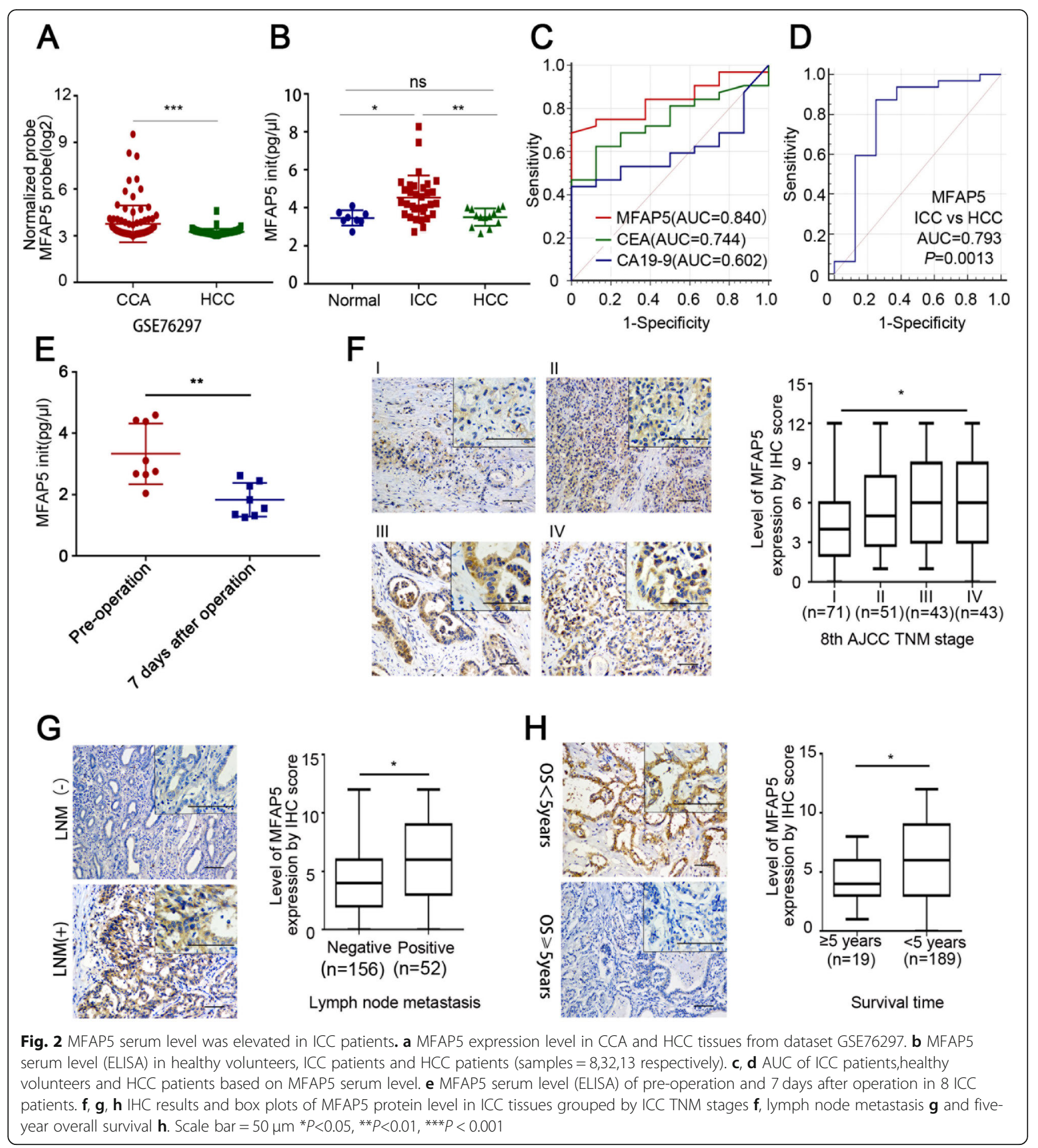

\section{Cell culture and transfection}

Human ICC cell lines RBE and SSP-25 were purchased from the Cell Resources Center of Shanghai Institutes for Biological Science, Chinese Academy of Science (Shanghai, China). Cells were cultured in RPMI-1640 (Gibco BRL, Rockville, MD, USA) and supplemented with $10 \%$ fetal bovine serum (Gibco BRL). For the gene knockdown assays, cells were infected with lentivirus encoding shRNA respectively. The target sequences used for MFAP5 shRNAs and Control shRNAs were listed in Additional file 1: Table S2.

\section{Western blot analysis}

Western blot analysis was performed as described previously [18]. The anti-MFAP5 antibody (\#ab283028) and anti-Ki67(\#ab156956) antibody were purchased from 
Abcam. The anti- $\beta$-actin (\#4970), anti-rabbit IgG, HRPlinked antibody (\#7071) and Notch activated targets antibody sample kit (\#68309) were obtained from Cell Signaling Technology. The anti-CCND1 (60186-1-Ig), anti-CDK4 (11026-1-AP), anti-CDK6 (14052-1-AP), anti-CDKN1A (10355-1-AP) and anti-CDC25A (550311-AP) antibodies were purchased from Maygene Co. The anti-NOTCH2(cleaved Ala1734) (\#PA5-37433) antibody was purchased from Thermo Fisher Scientific.

\section{Co-immunoprecipitation (co-IP)}

$2 \times 10^{7}$ RBE and SSP-25 PBS-washed cells were harvested and lysed with Pierce lysis buffer (Thermo, cat\#8990) containing protease inhibitors cocktail (TargetMol, cat\#C0001). Then incubated with NOTCH1 (CST,cat\#3608), MFAP5 (abcam,ab203828) antibody and control IgG (CST,cat\#3900) after centrifugation respectively. Subsequently, the cell lysates were incubated with Protein A/G Dynabeads (Thermo, cat\#10002D/10004D). Afterwards, the protein A/G Dynabeads were eluted and collected. The eluent was boiled and denatured for Western-Bolt.

\section{Assay for transposase accessible chromatin with high- throughput sequencing}

Chromatin preparation Nuclei was prepared from $4 \times$ $10^{4}$ cells. Library amplification was performed using the NEBnext High Fidelity 2× PCR Master Mix (\#M0541S, New England Biolabs) according to previously published PCR conditions. ATAC-seq library preparations were sequenced using single-end 50-bp reads on the Illumina HiSeq 2000 platform. Raw reads were adaptor-trimmed using Trim Galore (v0.2.5) and aligned to the genome with Bowtie (v1.0.1) with the $\mathrm{m} 1$ option enabled to allow only uniquely aligned high-quality reads. Peaks were called using the MACS2 software (v2.1.0.20140616) with the options -q 0.05 to retain significant peaks and shift size 50 to account for the transposase fingerprint, while default parameters were used for other options.

\section{Statistical analysis}

Statistical analyses were carried out by SPSS software 24.0 and GraphPad Prism 7.0 (La Jolla, CA, USA). Results were presented as mean \pm standard deviation (SD). Student t-test (paired/unpaired) was used for values following normal distribution. Non-parametric data were compared using the Mann-Whitney $U$ test and Wilcoxon test. Chi-Square test was used for testing the differences between categorical variables. Fisher's exact test was used when the number of variables was lower than 5. Correlation analysis were performed using the Spearman correlation tests. The survival curves were obtained by Kaplan-Meier method and compared using the logrank test. The optimal cut-off value of continuous variables was determined by ROC curve analysis. The DeLong test was used to compare the difference between the AUCs of different biomarkers [19]. The ANOVA test was performed to compare the mean values of proliferation rate in different groups. It was considered to be statistically different when $P<0.05$ at two-tail (" $P<0.05$, ${ }^{* *} P<0.01$, ${ }^{* * * *} P<0.001$, $\left.{ }^{* * * *} P<0.0001\right)$.

\section{Results}

MFAP5 expression was upregulated in ICC patients and correlated with poor prognosis

To determine the functional and clinical relevance of ECM-related genes in ICC, we first analyzed all the differentially expressed protein-coding genes within two microarray datasets (GSE76297 and GSE26566) from the GEO database. Among the seven different probes that showed consistent results in the two datasets, MFAP5 was the most significantly upregulated ECM gene in both datasets (Figs. 1a, b, Additional file 1: Figure S1a). The expression level of MFAP5 differed statistically between tumor and para-tumor tissues (Additional file 1: Figure S1b, c). To validate these findings, we evaluated the expression level of MFAP5 in a cohort of 24 pairs of ICC and para-tumor (non-cancerous) tissues. The results showed that MFAP5 mRNA expression level was significantly higher in ICC tissues than in para-tumor tissues (Fig. 1C). IHC analysis showed that positive staining of the MFAP5 protein was enriched in ICC tissues, but was rarely observed in para-tumor and normal tissues (Fig. 1d). The results also showed that MFAP5 expression was significantly higher in ICC tissues than in non-cancerous tissues. Furthermore, to investigate the effect of MFAP5 expression on postoperative survival in ICC patients, we utilized univariate statistical methods to analyze clinical data from 208 ICC patients. The univariate and multivariate analysis showed that MFAP5 protein level, tumor vascular invasion, lymph node metastasis, and tumor differentiation were prognostic factors for disease-free survival (DFS) and overall survival (OS) (Table 1). Patients were divided into two groups based on the optimal level of MFAP5, namely, highexpression group (MFAP5 $>4, n=107)$ and lowexpression group (MFAP $5 \leq 4, n=101$ ). Both of OS and DFS were significantly different between the two groups (Fig. 1e, f). These results suggested that the MFAP5 gene might play an important role in ICC progression.

\section{MFAP5 serum level was elevated in ICC patients}

Analysis of the GSE76297 dataset showed that there was a significant difference in MFAP5 expression between CCA and HCC patients (Fig. 2a). To test whether MFAP5 could be used as an early diagnostic serum index to discriminate ICC from HCC, we performed an exploratory analysis of MFAP5 serum level in a cohort 
Table 1 Prognostic factor for DFS and OS of patients with intrahepatic cholangiocarcinoma determined by using univariate

\begin{tabular}{|c|c|c|c|c|c|c|}
\hline \multirow[t]{2}{*}{ Variable } & \multicolumn{3}{|c|}{ Disease-free survival } & \multicolumn{3}{|c|}{ Overall survival } \\
\hline & $\mathrm{HR}$ & $95 \% \mathrm{Cl}$ & $P$-value & $\mathrm{HR}$ & $95 \% \mathrm{Cl}$ & $P$-value \\
\hline \multicolumn{7}{|l|}{ Univariate } \\
\hline Age $(>58$ or $\leq 58)$ & 0.95 & $0.68-1.33$ & 0.571 & 1.01 & $0.72-1.41$ & 0.945 \\
\hline Gender (female/male) & 1.23 & $0.88-1.72$ & 0.201 & 1.23 & $0.88-1.72$ & 0.208 \\
\hline Hepatitis B (-/+) & 0.94 & $0.62-1.43$ & 0.788 & 0.88 & $0.58-1.32$ & 0.547 \\
\hline Alb $>40 \mathrm{~g} / \mathrm{L}$ or $\leq 40 \mathrm{~g} / \mathrm{L}$ & 0.88 & $0.63-1.23$ & 0.455 & 0.85 & $0.61-1.18$ & 0.329 \\
\hline Hepatitis C (+/-) & 1.75 & $0.54-5.62$ & 0.192 & 1.75 & $0.54-5.64$ & 0.199 \\
\hline MFAP5 protein level $(>4 / \leq 4)^{a}$ & 1.76 & $1.25-2.47$ & 0.0004 & 1.71 & $1.22-2.40$ & 0.001 \\
\hline CA19-9(>37 U/L or $\leq 37 \mathrm{U} / \mathrm{L})$ & 1.70 & $1.22-2.38$ & 0.031 & 1.91 & $1.37-2.66$ & 0.043 \\
\hline $\mathrm{CEA}(>5 \mu \mathrm{g} / \mathrm{L}$ or $\leq 5 \mu \mathrm{g} / \mathrm{L})$ & 1.76 & $1.21-2.55$ & 0.011 & 1.84 & $1.27-2.67$ & 0.017 \\
\hline Vascular invasion (+/-) & 2.32 & $1.36-3.95$ & 0.0001 & 2.54 & $1.46-4.41$ & 0.0001 \\
\hline Number of tumor (multiple/single) & 1.79 & $1.19-2.68$ & 0.0006 & 1.85 & $1.23-2.78$ & 0.0003 \\
\hline Lymph node metastasis (+/-) & 1.48 & $1.03-2.12$ & 0.017 & 1.40 & $0.98-1.99$ & 0.047 \\
\hline Adjacent organ invasion (+/-) & 1.21 & $0.82-1.78$ & 0.284 & 1.29 & $0.87-1.91$ & 0.160 \\
\hline \multicolumn{7}{|l|}{ Tumor differentiation } \\
\hline Well vs Moderately & 0.44 & $0.22-0.91$ & 0.095 & 0.38 & $0.19-0.74$ & 0.041 \\
\hline Well vs Poorly & 0.30 & $0.15-0.59$ & 0.009 & 0.27 & $0.14-0.51$ & 0.002 \\
\hline Moderately vs Poorly & 0.60 & $0.41-0.88$ & 0.002 & 0.61 & $0.42-0.90$ & 0.005 \\
\hline \multicolumn{7}{|l|}{ Multivariate } \\
\hline${ }^{\mathrm{a} M F A P 5}$ protein level $(>4 / \leq 4)$ & 1.88 & $1.29-2.75$ & 0.0008 & 1.75 & $1.20-2.56$ & 0.003 \\
\hline Vascular invasion (+/-) & 1.72 & $1.12-2.65$ & 0.012 & 1.77 & $1.15-2.79$ & 0.008 \\
\hline Lymph node metastasis (+/-) & 1.93 & $1.29-2.87$ & 0.001 & 1.55 & $1.04-2.32$ & 0.028 \\
\hline Tumor differentiation (Well vs Moderately vs Poorly) & 1.55 & $1.25-1.93$ & 0.0001 & 1.38 & $1.12-1.69$ & 0.002 \\
\hline
\end{tabular}

MFAP5 Microfibril associated protein 5, CA19-9 Carbohydrate antigen 19-9, CEA Carcino-embryonic antigen, DFS Disease-free survival, OS, Overall survival, HR Hazard ratio, $\mathrm{Cl}$ Confidence interval

${ }^{\mathrm{a}}$ Immunohistochemical (IHC) score, split at median

of 32 ICC patients and $13 \mathrm{HCC}$ patients. For the control, we measured MFAP5 serum level in healthy volunteers who had healthy medical reports. Analysis in this exploratory cohort revealed significantly elevated MFAP5 level in ICC patients' serum samples compared to serum samples from healthy volunteers. Importantly, ICC patients also showed significantly higher serum MFAP5 level compared to HCC patients (Fig. 2b). Based on the elevated MFAP5 expression level in serum samples from the cohort of ICC patients, we next evaluated the diagnostic power of serum MFAP5 as a diagnostic marker for ICC by performing ROC curve analysis. The analysis revealed an AUC of 0.840 for the differentiation between healthy volunteers and ICC patients based on their initial MFAP5 serum level. The diagnostic power of initial serum MFAP5 was superior to initial CEA and CA19-9 serum level, which showed an AUC of 0.744 and 0.602 respectively (Fig. 2c). Arguing for a specific elevation of serum MFAP5 level between ICC and HCC patients, the ROC curve analysis revealed an AUC of 0.793 for the differentiation of HCC and ICC patients (Fig. 2d). To test whether MFAP5 could be used as a biomarker for
ICC therapies, we performed an analysis of MFAP5 serum level in a cohort of 8 ICC patients. Each case included one sample of pre-operation and one sample of 7 days after operation. We tested MFAP5 serum level by ELISA and analyzed the data with the Paired-Sample T Test. The results showed that MFAP5 serum level was significantly higher in preoperative serum than in postoperative serum $(P=0.0031$; Fig. $2 \mathrm{e})$. This indicated that MFAP5 might be used as a biomarker for evaluating the efficiency of therapies of ICC.

To further investigate the role of MFAP5 in the aggressive progression of ICC, we explored the correlation of MFAP5 expression and the clinicopathologic characteristics of 208 ICC patients. As shown in Table 2, MFAP5 level in higher ICC TNM stages were higher than those in low ICC TNM stages (Sample numbers of stages I, II, III, IV were 71, 51, 43, 43; $P=0.0141$; Fig. 2f), indicating a correlation between MFAP5 expression and ICC TNM stages. MFAP5 level was significantly higher in ICC cases exhibiting positive lymph node metastasis (LNM) than in those without LNM (Sample numbers of Negative and Positive LNM were 156 and 
Table 2 Correlation between MFAP5 expression and intrahepatic cholangiocarcinoma in 208 ICC patients

\begin{tabular}{|c|c|c|c|}
\hline \multirow[t]{2}{*}{ Characteristics } & \multicolumn{2}{|l|}{ Number of patients } & \multirow{2}{*}{$\begin{array}{l}\text { P- } \\
\text { value }^{a}\end{array}$} \\
\hline & High MFAP5 expression & Low MFAP5 expression & \\
\hline Gender & & & 1.000 \\
\hline Female & 49 & 46 & \\
\hline Male & 58 & 55 & \\
\hline Age & & & 0.581 \\
\hline$>58$ & 53 & 46 & \\
\hline$\leq 58$ & 54 & 55 & \\
\hline Tumor & & & 0.7815 \\
\hline$>5 \mathrm{~cm}$ & 55 & 49 & \\
\hline$\leq 5 \mathrm{~cm}$ & 52 & 52 & \\
\hline Tumor number & & & 0.473 \\
\hline single & 69 & 77 & \\
\hline Multiple & 38 & 34 & \\
\hline Tumor differentiation & & & 0.477 \\
\hline Well & $4^{b}$ & 5 & \\
\hline Moderate & 64 & 67 & \\
\hline Poor-undifferentiated & 39 & 29 & \\
\hline Vascular invasion & & & 0.720 \\
\hline Not present & 86 & 84 & \\
\hline Present & 21 & 17 & \\
\hline Lymph node invasion & & & 0.0001 \\
\hline Not present & 67 & 89 & \\
\hline Present & 40 & 12 & \\
\hline AJCC 8th TNM stage & & & 0.006 \\
\hline । & 35 & 31 & \\
\hline$\|$ & 17 & 35 & \\
\hline III & 27 & 16 & \\
\hline IV & 28 & 15 & \\
\hline 3-year survival & & & 0.058 \\
\hline$\geq 36$ months & 16 & 26 & \\
\hline$<36$ months & 91 & 75 & \\
\hline 5-year survival rate & & & 0.030 \\
\hline$\geq 60$ months & 5 & 14 & \\
\hline$<60$ months & 102 & 87 & \\
\hline
\end{tabular}

${ }^{a}$ Chi-square test

${ }^{\text {b}}$ Fisher's exact test

52; $P=0.0189$; Fig. 2g). Similarly, patients survived more than five years after surgery had significantly lower MFAP5 IHC scores than patients who died within five years (Sample numbers of $>=5$ years and $<5$ years OS were 19 and 189; $P=0.0274$; Fig. 2h).

\section{MFAP5 promoted ICC cells proliferation both in vitro and} in vivo

To investigate the function of MFAP5 in the progression of ICC, RBE and SSP-25 human ICC cell lines were co- cultured with purified recombinant MFAP5 protein (recMFAP5). The results demonstrated that exogenous recMFAP5 increased ICC cells' proliferation in a dosedependent manner (Fig. 3a). We also established stable MFAP5 knockdown RBE and SSP-25 ICC cells with two respective MFAP5 shRNAs (Additional file 1: Figure S2a, b). The proliferation rate was significantly inhibited in MFAP5 knockdown RBE and SSP-25 cells compared to control cells (Fig. 3b). Moreover, colony-forming ability was markedly promoted by recMFAP5 in both RBE and 


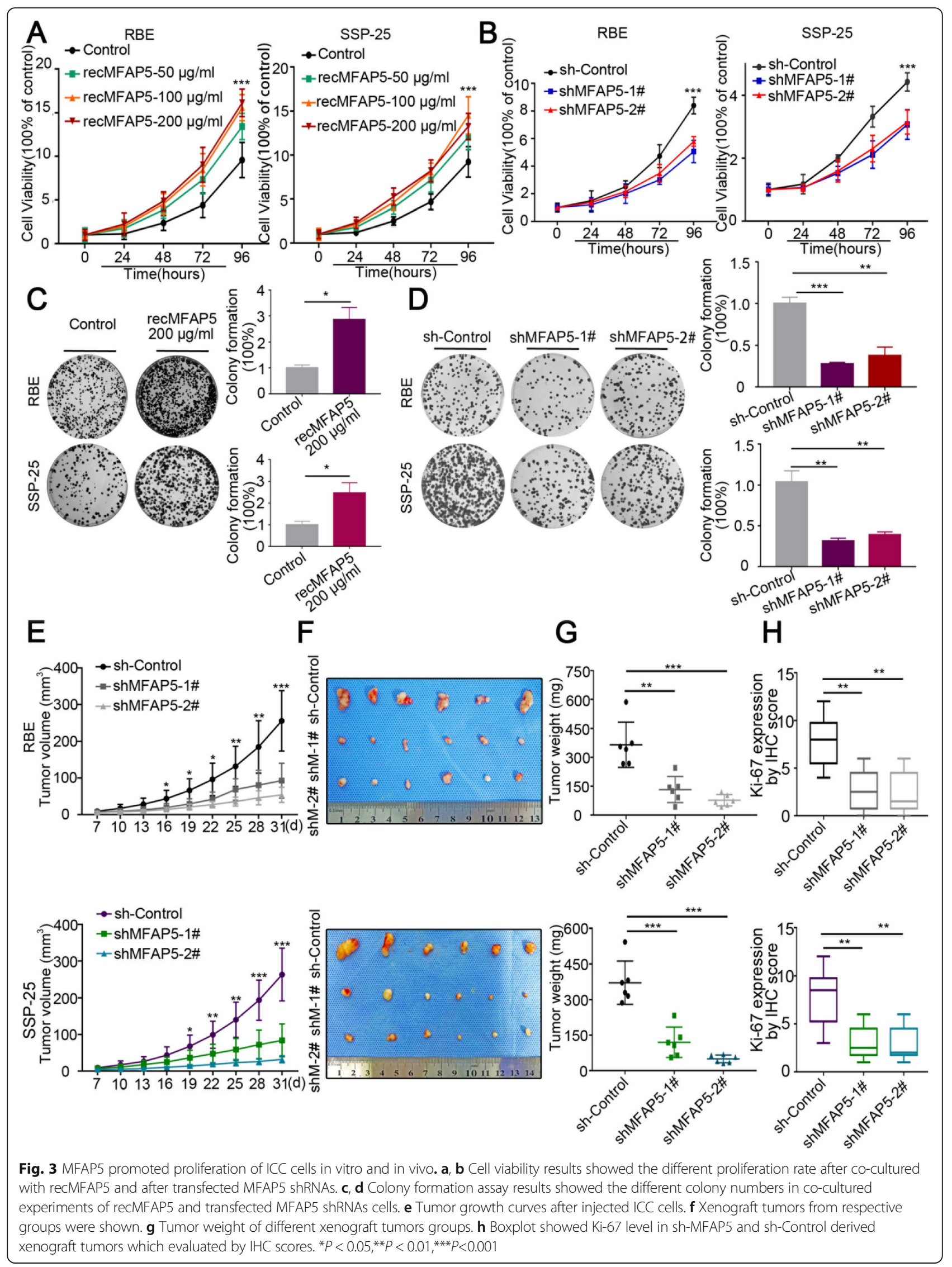


SSP-25 cells (Fig. 3c). In contrast, down-regulation of MFAP5 substantially suppressed colony formation in RBE and SSP-25 cells (Fig. 3d).

To evaluate whether the expression level of MFAP5 could affect ICC cell growth in vivo, we subcutaneously injected MFAP5 knockdown or control RBE and SSP-25 cells into nude mice. We observed that the tumor growth rate of MFAP5-silenced groups was markedly slower than that of the control groups for both RBE and SSP-25 cell lines (Fig. 3e). The mice were euthanized, and the subcutaneous tumors were measured every 3 days until 31 days after cell injection (Fig. 3f). The tumor weight was significantly lower in the MFAP5-silenced groups than in the control groups (Fig. 3g). Furthermore, IHC staining revealed that the expression of MFAP5 and Ki-67 were markedly downregulated in MFAP5 knockdown tumors (Figs. 3h, Additional file 1: Figure S2c-f).

\section{MFAP5 facilitated CCND1/CDK4/6/CDC25A-dependent G0/ G1 to S-phase transition}

To explore whether tumor growth promoted by MFAP5 is due to cell cycle acceleration, cell cycle analysis was performed after treating REB and SSP-25 cells with recMFAP5. Treatment with recMFAP5 decreased the fraction of cells in the G0/G1 phase and increased the fraction of cells in G2/M compared to the DMSO control (Fig. 4a, b). Addition of MFAP5 resulted in increased numbers of RBE cells in the $\mathrm{S}$ phase, but did not increase the proportion of S-phase SSP-25 cells; this may have been due to MFAP5-induced S-phase SSP-25 cells entering the G2/M phase. Similarly, MFAP5 silencing resulted in fewer cells in G2/M and an increase in the fraction of cells in the G0/G1 phase (Fig. 4c, d). However, the percentages of cells in the $S$ phase were not lower with MFAP5 knockdown. These results indicate that the ICC cells could be arrested at the G0/G1 phase via silencing of MFAP5.

We also investigated the mechanisms underlying the effects of MFAP5 on the G0/G1 to S-phase transition. Given that CDK4/6/CDC25A are important for the G0/ G1 to $\mathrm{S}$ transition during the cell cycle, we postulated that CDK4/6/CDC25A may participate in MFAP5mediated cell cycle regulation. This hypothesis was supported by the data showing that recMFAP5 treatment increased CCND1/CDK4/6/CDC25A expression but reduced p21 expression in ICC cells (Fig. 4e). In addition, CCND1/CDK4/6/CDC25A protein and mRNA levels were significantly attenuated by MFAP5 knockdown, whereas p21 expression was increased (Fig. 4f, g, h). These results suggest that MFAP5 may increase CCND1/CDK4/6/CDC25A transcription by inhibiting p21 activity and thus promote G0/G1 to S-phase transition and cell proliferation.
MFAP5 positively regulated the transcription of Notch1 pathway genes

To further characterize the regulatory effect of MFAP5 on the cell cycle and cell proliferation, the transcription status of ICC cells transduced by MFAP5 shRNA or control shRNA (shControl) was compared using RNAseq (Figs. 5a, Additional file 1: Figure S3a, 3b). The gene set enrichment analysis (GSEA) showed that the mRNA levels of Notch signaling pathway genes were markedly reduced with MFAP5 silencing (Fig. 5b, c). We next validated the regulatory effect of MFAP5 on components of the Notch signaling pathway. Western blot and qPCR analysis showed that several Notch signaling pathway components, as well as Notch signaling targets, were reduced in MFAP5 knockdown cells compared to shControl cells (Fig. 5d, e). Additionally, shMFAP5 treatment resulted in significant repression of the NOTCH1 intracellular domain (NICD1), MAML1, and HES1, but there was no significant difference in $\mathrm{NOTCH} 2$ intracellular domain (NICD2) (Additional file 1: Figure S3c, d). These results suggest that MFAP5 may interact directly with the NOTCH1 receptor to activate the Notch1 signaling pathway. To further confirm the regulatory role of MFAP5 on Notch1 signaling, the expression of these Notch1 signaling factors was compared between recMFAP5- and DMSO-treated cells using western blotting. Strong positive correlations were observed between recMFAP5 dosage and the expression of Notch1 signaling factors (Figs. 5f, g, Additional file 1: Figure S3e). These findings demonstrated that MFAP5 plays important roles in regulating the Notch1 signaling pathway. In order to explore how MFAP5 regulated Notch1 pathway, we performed the Co-IP experiment in ICC cell lines (RBE and SSP-25). The results showed that MFAP5 could bind directly with NOTCH1, indicating that MFAP5 regulated Notch1 pathway by interacting directly with Notch1 receptor (Fig. 5h, i).

\section{Chromatin accessibility by ATAC-seq analysis revealed a role for MFAP5 in Notch1 activation}

As the Notch1 signaling pathway was shown to be involved in MFAP5-mediated ICC aggressiveness, we next investigated the effect of the NOTCH inhibitor FLI-06 on recMFAP5-induced cell outgrowth. Interestingly, the accelerated proliferation of ICC cells induced by MFAP5 was significantly inhibited in the presence of FLI-06 (Fig. $6 a, b)$. Further analysis revealed that FLI-06 could effectively abolish the MFAP5-induced overexpression of CCND1, CDK6, CDKN1A, and MYC (Fig. 6c). These findings indicate that the Notch1 pathway is important for MFAP5-enhanced ICC cell growth.

To further identify the target factors regulated by the MFAP5/Notch1 axis, genome-wide chromatin accessibility was assessed by Assay for Transposase-Accessible 


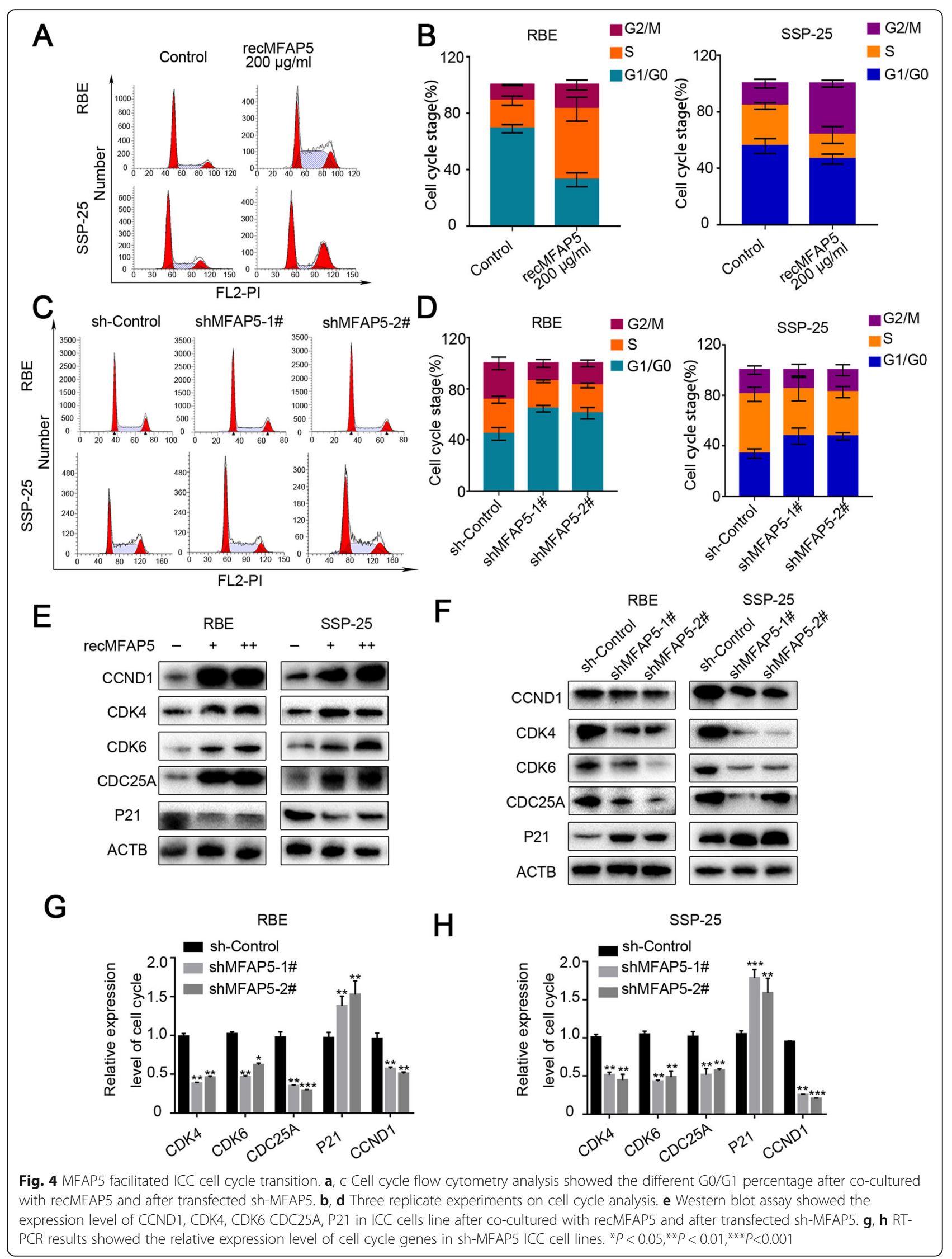




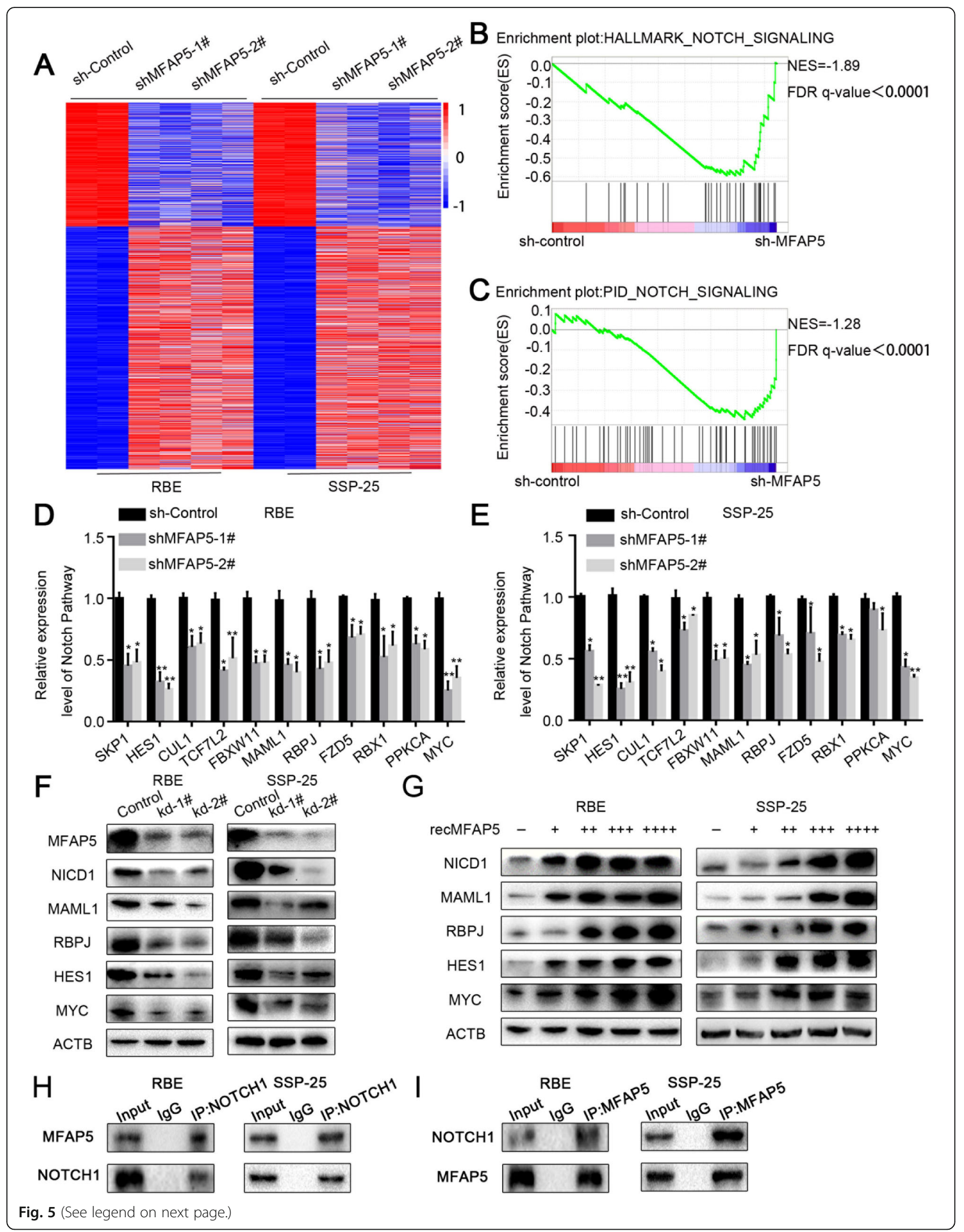


(See figure on previous page.)

Fig. 5 MFAP5 positively regulated Notch1 pathway transcription. a Heat map showed results of RNA-seq of SSP-25 and RBE transformed shcontrol, sh-MFAP5-1\# and sh-MFAP5-2\#. b, c GSEA results showed down-regulation of Notch pathway in cells transfected sh-MFAP5 compared with sh-Control. $\mathbf{d}$, e RT-PCR results showed the mRNA level of genes in NOTCH1 pathway after transfected sh-MFAP5 in ICC cell lines. $\mathbf{f}, \mathbf{g}$ Western blot analysis of Notch1 pathway target genes in sh-MFAP5 and sh-Control cells (f) and co-culture with recMFAP5 and control cells (G, each " + " represented 50 $\mathrm{mg} / \mathrm{ml}$ recMFAP5). $\mathbf{h}, \mathbf{i}$ Co-IP results showed that MFAP5 could bind directly with NOTCH1. ${ }^{*} P<0.05$, ${ }^{* *} P<0.01$, ${ }^{* * *} P<0.001$

Chromatin using sequencing (ATAC-seq). There were significant changes in chromatin architecture with appearing peaks associated with upregulated gene expression in recMFAP5-treated ICCs compared to the DMSO control (Fig. 6d). Surprisingly, many appearing peaks in recMFAP5-treated ICCs were absent in ICCs co-treated with recMFAP5 and FLI-06. We interpret this finding to mean that FLI-06 suppressed the downstream transcription events induced by MFAP5/Notch1 signaling. The results of the GSEA analysis showed an enrichment of genes assigned to the cell cycle gene ontology (GO) term that were inactivated in FLI-06-treated ICCs and significantly upregulated in ICCs treated with recMFAP5 (Fig. 6e). Figure $6 \mathrm{f}$ shows representative examples of these target genes, including CCND1, $C D C 25 A$, and CDK6. The results also revealed significant changes in chromatin architecture, with disappearing peaks associated with FLI-06 co-culture.

\section{Discussion}

The ECM regulates tissue homeostasis, and ECM dysregulation contributes to tumor progression. The ECM factors within an abnormally remodeled ECM can enhance ICC tumor progression and aggressiveness. We demonstrated here that MFAP5 is highly expressed in the ECM of ICC and is correlated with worse outcome. MFAP5 secreted by ICC tumors enters the blood of patients and can be detected specifically and sensitively by ELISA experiments. MFAP5 in the ECM enhanced the activation of the Notch1 pathway, facilitating downstream gene transcription and thereby promoting G1 to $\mathrm{S}$ cell cycle phase transition as well as proliferation (Fig. $6 \mathrm{~g}$ ). Given the lack of effective early ICC diagnosis methods and the difficulty in distinguishing ICC from HCC in the early stages of the diseases, our results demonstrated that MFAP5 serological detection may be used as an effective method for early ICC diagnosis and for evaluating the efficiency of therapies; in turn, MFAP5 inhibition would likely be used as a treatment. Our findings also revealed an important mechanism underlying amplified Notch activation in ICC that is mediated by MFAP5 in the tumor microenvironment.

Identification of specific ICC diagnostic or prognostic biomarkers is urgently required. To date, few ideal screening analyses have been developed. Analysis of the CA19-9 biomarker may aid in CCA diagnosis, but the levels of CA19-9 in ICC patients are heterogeneous. Li et al. defined a biliary vesicle miR-based panel that can be used for CCA diagnosis [20], while Andresen et al. identified DNA methylation of CDO1, CNRIP1, SEPT9, and VIM displaying frequencies of $45-77 \%$ in biliary brushes from CCA patients [21]. Anti-glycoprotein 2 (anti-GP2) has also been reported as a potential diagnostic biomarker in CCA as well as in secondary sclerosing cholangitis (SSC) [22]. However, none of these biomarkers can effectively distinguish HCC from ICC, two diseases that require different surgical and postoperative drug treatments [4]. Serum metabolites have also been used as diagnostic biomarkers for CCA and HCC. These findings indicated integration of genomics, transcriptomics, and metabolomics for the identification of HCC and ICC subtypes. In our study, MFAP5 levels did not increase in the serum of either healthy volunteers or HCC patients, but increased specifically in the serum of ICC patients. The specificity and sensitivity of MFAP5 are higher than those of traditional biomarkers (e.g., CEA and CA19-9). We also found that the serum level of MFAP5 was significantly decreased in the serum of post-operation compared with the serum of preoperation. This indicated that MFAP5 might be used as a biomarker for evaluating the efficiency of therapies of ICC. Importantly, in this study, MFAP5 was correlated with various malignant indexes (e.g., metastasis and poor OS) and can therefore be used as a prognostic marker in patients with ICC.

The cancer microenvironment contains both cellular and non-cellular components, including the extracellular matrix, and is critical for the activation of tumor survival signals. Although Notch signaling has previously been reported to regulate liver metabolism, inflammation, and cancer, the interaction between the composition in an ECM context and Notch signaling remain largely unknown [23]. A recent study has identified the interaction between the ECM and integrin $\alpha 5$ as the extracellular cue that activates Notch signaling in pancreatic progenitors via the F-actin-YAP1-Notch axis [24]. Notch signaling promotes ECM remodeling during endocardial projection formation, providing new insights into the pathology of congenital heart disease [25]. In our study, we showed that a NOTCH1 inhibitor phenocopied MFAP5 knockdown and abolished MFAP5-induced ICC outgrowth, strongly suggesting that MFAP5 acts upstream of Notch signaling in ICC. NICD1 levels increased in a dose-dependent manner in ICC cells treated 


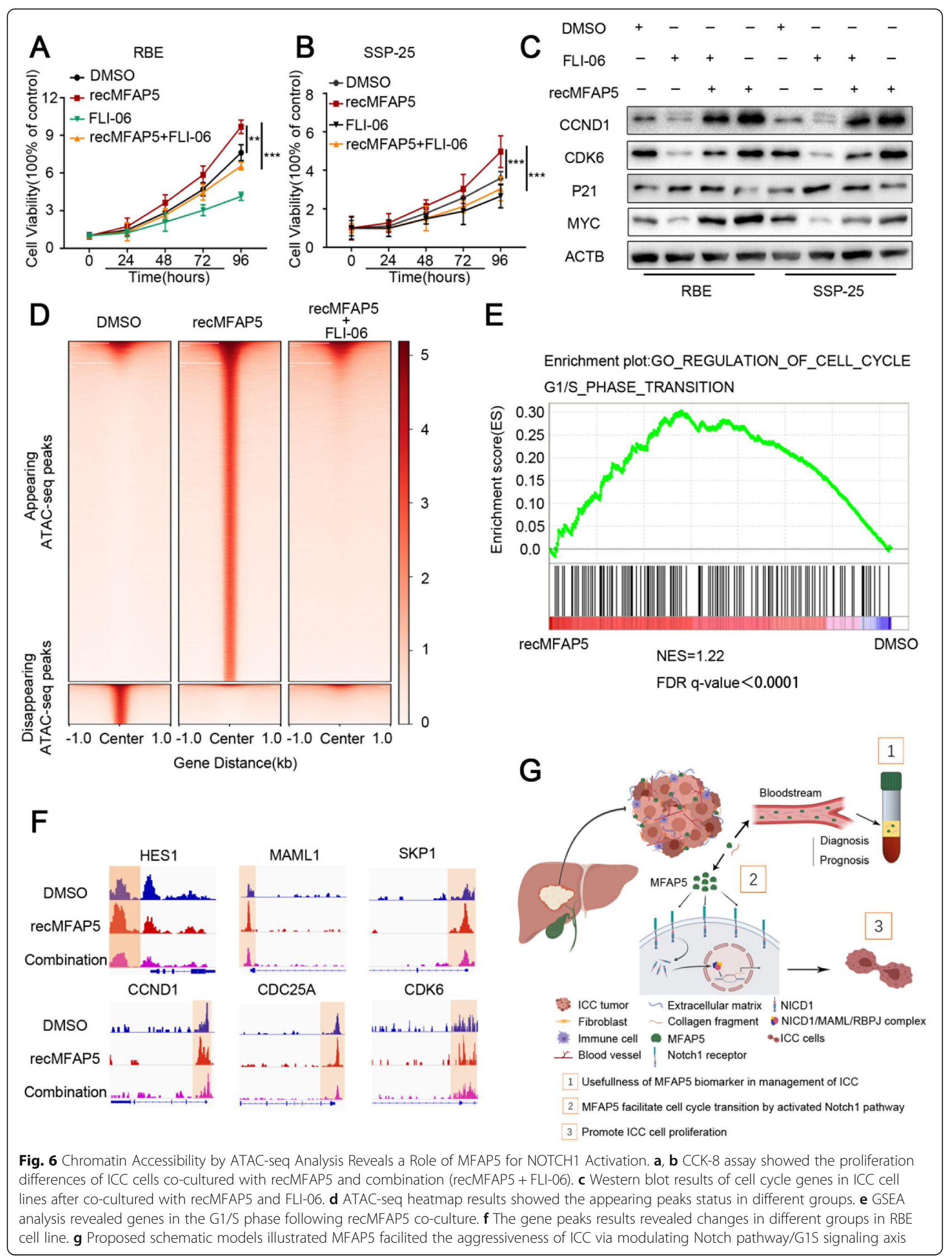


with MFAP5 and Co-IP results showed that MFAP5 could interact directly with Notch1 receptor, verifying that MFAP5 acts upstream of Notch1 signaling in ICC. Activated Notch1 signaling elicited increased transcription of downstream genes that are reported to promote ECM remodeling in ICC. Further context-specific understanding of the MFAP5/Notch1 signaling axis in ICC will be essential to translate these findings into clinical practice.

Cell-cycle regulation by intracellular molecular pathways has been extensively studied. Additionally, the cell cycle is also regulated via cell-cell physical forces and cell-ECM interfaces [26]. Early studies established that cell shape, polarity, and adhesion strongly influence DNA synthesis and cell growth. However, substantially less is known about the mechanical regulation of the cell cycle by the ECM. Previous studies showed that MFAP5 inhibition induces G2/M phase arrest, decreases the expression of Cyclin B1, Cyclin D1, and CDK4, and enhances p21 and p53 levels in cervical cancer. Inhibition of cell growth by MFAP5 knockdown is dependent on reactive oxygen species (ROS) production [27]. In our study, we showed that MFAP5 promotes ICC G0/G1 to S-phase cell cycle transition which is dependent on Notch1 signaling activation. The Notch1 signaling pathway was reported to enhance the expression of the cyclin E protein in cholangiocellular carcinomas [28]. However, we did not observe any change in cyclin E expression, either with overexpression or with knockdown of MFAP5 (data not shown). In contrast, the levels of CCND1 and CDK4/6, regulators of the G0/S checkpoint, were significantly upregulated though MFAP5/Notch1 activation. In agreement with this, significant changes in chromatin architecture with appearing peaks were associated with upregulated CCND1 and CDK6 gene expression.

\section{Conclusions}

In conclusion, our results unveil serum MFAP5 as a potential diagnostic, prognostic and therapeutic tool for ICC, providing a novel approach for use in noninvasive screening of ICC. ECM-derived MFAP5 functions as an oncogenic protein that may participate in Notch1 signaling activation in ICC. The mode and mechanism of the suppression of tumor aggressiveness by MFAP5 depletion are potentially important. Consequently, large-scale studies should be undertaken to evaluate the therapeutic potential of MFAP5 in ICC.

\section{Supplementary information}

Supplementary information accompanies this paper at https://doi.org/10. 1186/s13046-019-1477-4.

Additional file 1: Figure S1. MFAP5 expression was upregulated in cholangiocarcinoma (CCA) patients by analysis GSE26566 and GSE 76297 datasets. Related to Fig. 1. Figure S2. Depletion of MFAP5 secretion inhibits ICC tumor growth in vivo. Related to Fig. 3. Figure S3. Silencing MFAP5 suppresses the expression of HES1 and MYC in ICC cells. Related to Fig. 5. Table S1. The clinic pathological characteristics of 208 ICC patients. Table S2. Sequences of primers and shRNAs used in this study.

\section{Abbreviations}

ATAC-seq: Assay for Transposase-Accessible Chromatin using sequencing; CA19-9: Carbohydrate Antigen 19-9; CCA: Cholangiocarcinoma;

CEA: Carcino-Embryonic Antigen; DFS: Disease-Free Survival;

ECM: Extracellular Matrix; ICC: Intrahepatic Cholangiocarcinoma; MFAP5: Microfibril Associated Protein 5; OS: OS overall survival; RNAseq: Whole Transcriptome Shotgun sequencing

\section{Acknowledgements}

Not applicable.

\section{Authors' contributions}

$J H L, X X Z$ and $X Y Y$ were involved in experiment design, acquisition of data, analysis and interpretation of data, and drafting of the manuscript. FXL and ZXG were involved in acquisition of ATAC-seq data. CSH, XTH, SJL, JQW and QCX were involved in experiment design and acquisition of xenograft data. WZ and XYY were involved in study concept and design, analysis and interpretation of data, revision of the manuscript, fund raising, and study supervision.

\section{Funding}

This study was supported by grants from the National Natural Science Foundation of China (81772522, 81472261, 81572766, and 31771630), the Natural Science Foundation of Guangdong Province (2016A030313238, 2017A030312009, 2019A1515010686), the Science and Technology Development Projects of Guangzhou, Guangdong, China (201604020044), the Guangdong Innovative and Entrepreneurial Research Team Program (2016ZT06S029) and the China Postdoctoral Science Foundation (2016 M602588, 2108 M643325, 2108 M643327), and Shenzhen Science Foundation (KY20160309)

Availability of data and materials

All data generated or analyzed during this study are included in this published article and its supplementary information files.

Ethics approval and consent to participate

This study was approved by the Ethical Committee of the First Affiliated Hospital of Sun Yat-sen University and Institutional Animal Care and Use Committee (IACUC) Sun Yat-Sen University.

Consent for publication

Informed consent was obtained from all individual participants included in the study.

\section{Competing interests}

The authors declare that they have no competing interests.

Received: 24 September 2019 Accepted: 11 November 2019

Published online: 27 November 2019

References

1. Shaib YH, Davila JA, McGlynn K, El-Serag HB. Rising incidence of intrahepatic cholangiocarcinoma in the United States: a true increase? J Hepatol. 2004; 40(3):472-7.

2. Sirica AE, Gores GJ, Groopman JD, Selaru FM, Strazzabosco M, Wang XW, et al. Intrahepatic Cholangiocarcinoma: continuing challenges and translational advances. Hepatology. 2019;69(4):1803-15.

3. Blechacz B, Komuta M, Roskams T, Gores GJ. Clinical diagnosis and staging of cholangiocarcinoma. Nat Rev Gastroenterol Hepatol. 2011;8(9):512-22.

4. Bridgewater J, Galle PR, Khan SA, Llovet JM, Park JW, Patel T, et al. Guidelines for the diagnosis and management of intrahepatic cholangiocarcinoma. J Hepatol. 2014;60(6):1268-89. 
5. Hyder O, Hatzaras I, Sotiropoulos GC, Paul A, Alexandrescu S, Marques H, et al. Recurrence after operative management of intrahepatic cholangiocarcinoma. Surgery. 2013;153(6):811-8.

6. Frangogiannis NG. Matricellular proteins in cardiac adaptation and disease. Physiol Rev. 2012;92(2):635-88.

7. Tlsty TD, Coussens LM. Tumor stroma and regulation of cancer development. Annu Rev Pathol. 2006;1:119-50.

8. Rizvi S, Gores GJ. Pathogenesis, diagnosis, and management of cholangiocarcinoma. Gastroenterology. 2013;145(6):1215-29.

9. Barbier M, Gross MS, Aubart M, Hanna N, Kessler K, Guo DC, et al. MFAP5 loss-of-function mutations underscore the involvement of matrix alteration in the pathogenesis of familial thoracic aortic aneurysms and dissections. Am J Hum Genet. 2014;95(6):736-43.

10. Principe S, Mejia-Guerrero S, Ignatchenko V, Sinha A, Ignatchenko A, Shi W, et al. Proteomic analysis of Cancer-associated fibroblasts reveals a paracrine role for MFAP5 in human Oral tongue squamous cell carcinoma. J Proteome Res. 2018;17(6):2045-59.

11. Wu Z, Wang T, Fang M, Huang W, Sun Z, Xiao J, et al. MFAP5 promotes tumor progression and bone metastasis by regulating ERK MMP signaling pathways in breast cancer. Biochem Biophys Res Commun. 2018:498(3):495-501.

12. Leung CS, Yeung TL, Yip KP, Wong KK, Ho SY, Mangala LS, et al. Cancerassociated fibroblasts regulate endothelial adhesion protein LPP to promote ovarian cancer chemoresistance. J Clin Invest. 2018;128(2):589-606.

13. Leung CS, Yeung TL, Yip KP, Pradeep S, Balasubramanian L, Liu J, et al. Calcium-dependent FAK/CREB/TNNC1 signalling mediates the effect of stromal MFAP5 on ovarian cancer metastatic potential. Nat Commun. 2014;5:5092.

14. Marti P, Stein C, Blumer T, Abraham Y, Dill MT, Pikiolek M, et al. YAP promotes proliferation, chemoresistance, and angiogenesis in human cholangiocarcinoma through TEAD transcription factors. Hepatology. 2015; 62(5):1497-510.

15. Huang XT, Huang CS, Li JH, Cai JP, Chen W, Yin XY. Prognostic significance of neutrophil/prealbumin ratio for intrahepatic cholangiocarcinoma undergoing curative resection. HPB (Oxford). 2018;20(12):1215-22.

16. Huang CS, Chu J, Zhu XX, Li JH, Huang XT, Cai JP, et al. The C/ EBPbeta-LINC01133 axis promotes cell proliferation in pancreatic ductal adenocarcinoma through upregulation of CCNG1. Cancer Lett. 2018;421: 63-72.

17. Hao XY, Cai JP, Liu X, Chen W, Hou X, Chen D, et al. EYA4 gene functions as a prognostic marker and inhibits the growth of intrahepatic cholangiocarcinoma. Chin J Cancer. 2016;35(1):70.

18. Mo SJ, Liu X, Hao XY, Chen W, Zhang KS, Cai JP, et al. EYA4 functions as tumor suppressor gene and prognostic marker in pancreatic ductal adenocarcinoma through beta-catenin/ID2 pathway. Cancer Lett. 2016; 380(2):403-12

19. DeLong ER, DeLong DM, Clarke-Pearson DL. Comparing the areas under two or more correlated receiver operating characteristic curves: a nonparametric approach. Biometrics. 1988;44(3):837-45.

20. Li L, Masica D, Ishida M, Tomuleasa C, Umegaki S, Kalloo AN, et al. Human bile contains microRNA-laden extracellular vesicles that can be used for cholangiocarcinoma diagnosis. Hepatology. 2014;60(3):896-907.

21. Andresen K, Boberg KM, Vedeld HM, Honne H, Jebsen P, Hektoen M, et al. Four DNA methylation biomarkers in biliary brush samples accurately identify the presence of cholangiocarcinoma. Hepatology. 2015;61(5):1651-9.

22. Ehlken H, Zenouzi R, Schramm C. Risk of cholangiocarcinoma in patients with primary sclerosing cholangitis: diagnosis and surveillance. Curr Opin Gastroenterol. 2017;33(2):78-84.

23. Morell CM, Strazzabosco M. Notch signaling and new therapeutic options in liver disease. J Hepatol. 2014;60(4):885-90.

24. Mamidi A, Prawiro C, Seymour PA, de Lichtenberg KH, Jackson A, Serup P, et al. Mechanosignalling via integrins directs fate decisions of pancreatic progenitors. Nature. 2018;564(7734):114-8

25. Luxan G, D'Amato G, MacGrogan D, de la Pompa JL. Endocardial notch signaling in cardiac development and disease. Circ Res. 2016;118(1):e1-e18.

26. Uroz M, Wistorf $S$, Serra-Picamal X, Conte V, Sales-Pardo M, Roca-Cusachs P, et al. Regulation of cell cycle progression by cell-cell and cell-matrix forces. Nat Cell Biol. 2018;20(6):646-54

27. Li Q, Zhang Y, Jiang Q. MFAP5 suppression inhibits migration/invasion, regulates cell cycle and induces apoptosis via promoting ROS production in cervical cancer. Biochem Biophys Res Commun. 2018;507(1-4):51-8.
28. Zender S, Nickeleit I, Wuestefeld T, Sorensen I, Dauch D, Bozko P, et al. A critical role for notch signaling in the formation of cholangiocellular carcinomas. Cancer Cell. 2013;23(6):784-95.

\section{Publisher's Note}

Springer Nature remains neutral with regard to jurisdictional claims in published maps and institutional affiliations.
Ready to submit your research? Choose BMC and benefit from:

- fast, convenient online submission

- thorough peer review by experienced researchers in your field

- rapid publication on acceptance

- support for research data, including large and complex data types

- gold Open Access which fosters wider collaboration and increased citations

- maximum visibility for your research: over $100 \mathrm{M}$ website views per year

At BMC, research is always in progress.

Learn more biomedcentral.com/submissions 